\title{
A Study of Treatment of Lizard Phobia Using Visible and Invisible Marker-based AR Application
}

\author{
1Zakiya A. Nayef, ${ }^{2}$ Hawar B. Ahmad \\ ${ }^{1}$ Computer Science Department, College of Computer Science \& I.T, Nawroz University, Kurdistan Iraq
}

\begin{abstract}
Augmented reality refers to the combination of computer-generated objects with real world environment. This technology is empowered by the evolution of hardware and software in the past decade. Virtual objects are placed through device screens in a user's environment relying on the device camera and capability of graphics hardware. This advancement opens a wide horizon for application development in the field of entertainment, education, medical, etc.

One of the main advantages of augmented reality (AR) is in the treatment of various phobias though exposure treatment. A phobia is characterized by irrational fear or reaction to a normal situation. Thus, gradually exposing patients to their phobias helps in reducing their uncontrolled reaction and aids in reducing their fear of their respective phobia. Herpetophobia is the extreme fear from aversion reptiles such as lizards. The lizard phobia is a common form of phobia amongst people.

In this article, an application of lizard phobia exposure treatment is proposed to help patients. The proposed application relies on marker-based AR. This is implemented using Visible marker AR system (VMARS) and Invisible marker AR system (IMARS), comparing the results of both systems to a group a subject to identify the best approach for Herpetophobia treatment.
\end{abstract}

Keywords: Augmented reality (AR), Virtual reality (VR), Phobia, Treatment, Visible_marker tracking.

\section{Introduction}

Mixed Reality computer interfaces refer to the combination of contents from the real world with virtual reality objects (Milgram and Kishino, 1994). Augmented Reality (AR) is part of the mixed reality computer interfaces it generates or projects virtual content into a user's perspective of the world. Nowadays, the concept of AR has integrated into graphical and audio contents. It usages have spread across different fields such as gaming industry, medical industry and education industry.

Augmented reality (AR) is a new technology in which various virtual elements incorporate into the user's perception of the real world. Phobia towards lizards

Academic Journal of Nawroz University

(AJNU) Volume 9, No 1 (2020).

Regular research paper: Published 4 March 2020

Corresponding author's e-mail : zakiyaduhoki87@gmail.com

Copyright (C2018 Zakiya Ali Nayef, Hawar B. Ahmad.

This is an open access article distributed under the Creative

Commons Attribution License. has been treated using exposure in vivo and virtual reality. Recently, augmented reality (AR) has also presented as a suitable tool. This proposal presents an augmented reality (AR) invisible marker-tracking system for the treatment of a phobia towards lizards. $A R$ refers to the introduction of virtual content into the real world, that is, the user is seeing an image composed of a real image and virtual elements superimposed over it. A phobia towards lizards has traditionally been treated using exposure in vivo and also using virtual reality (VR) (Hani Alkurdi, 2017).

In that work, visible markers use for tracking. In this proposal, we present a second version of the visiblemarker-tracking AR system (VMARS) in which markers that are invisible to the naked eye (invisiblemarkers) are used for tracking. The invisible marker-tracking AR system (IMARS) uses visiblemarkers that are drawn with a special ink. In 
visible-marker-tracking systems, users relate the markerwith the appearance of virtual elements. In our case, visible markers warn the patients of the appearance of lizards and this could produce an initial anxiety without the appearance of the virtual element that really should produce this anxiety. We consider the non-appearance of the markers to be Important, especially in some steps of the protocol followed during treatment. One common step of protocols for treating patients with phobia towards lizards is that patients have to search for the feared lizards in the same way they would do so in their house.

To simulate this work, we use three boxes, under one of which a marker is randomly placed. The lizards will appear over the marker. At first, when the patient sees part of the marker, the system still does not show the lizards, but the patient knows they will appear and she/he can decide not to finish raising the box, and the lizards will not appear. If the marker were not visible, the patient would not know that lizards are going to appear, and when they appear, they will produce the desired surprise. To avoid the one-to-one correspondence between the appearance of the marker and the appearance of lizards, it can be possible to pepper the environment with a large number of markers and just randomly make one active at any given time. But, in both cases, the markers appear artificially in the environment.

In this context of research proposal, there are few problems statements are needed to be address that the two systems, IMARS and VMARS, have problems associate with marker tracking: fast movements or changing light conditions cause tracking to fail, the entire marker must be in view for tracking to work, the problem of occluding the marker. Therefore, the primary hypothesis is the IMARS will induce a similar or higher sense of presence and will provoke a similar or higher level of anxiety than the VMARS with nonphobic participants. The aim of this study is to test if the IMARS induces a similar or higher sense of presence and provokes a similar or higher level of anxiety than the VMARS with non-phobic participants.

\section{System Background}

From types of phobia towards lizards become anxious when they are in a situation where these lizards can appear. They suffer an unrealistic and excessive fear that makes life miserable. They are always frightened of seeing the lizard they fear.

\subsection{Treatment of phobia towards small animals using virtual reality exposure therapy}

Virtual reality exposure therapy (VRET) uses VR as a tool for therapy. The therapist has significant control and can manipulate the virtual environments to some extent. In addition, VRET is based on the assumption that virtual environments can elicit anxiety and provide the opportunity for habituation (Rothbaum et al., 1995). In the DSM-IV, anxiety is defined as an "apprehensive anticipation of future danger or misfortune accompanied by a feeling of dysphonia or somatic symptoms of tension". This definition implies that anxiety is a future-oriented state, which causes the organism to behave so that the danger can be averted. Several studies support the idea that emotions such as fear or anxiety, which are felt during immersion, are indicators of the sense of presence in virtual environments (Robillard et al., 2003; Schuemie et al., 2000; Renaud et al., 2002; Regenbrecht, Schubert \&Friedmann, 1999; Bouchard et al., 2008). In (SusoRibera C., Fernández-Álvarez J., 2019) study aggregated data from three randomized control trials to explore the differential efficacy of three forms of exposure therapy, namely, in vivo (iVET), virtual reality (VRET), and augmented reality (ARET), in the treatment of small animal phobia. People who suffer 
(Hani Alkurdi, , 2017) used immersive VR for exposure therapy. The first experiment was carried out at the U.W. Human Interface Technology laboratory (HITLab). The first patient treated with this system needed 12 VR therapy sessions of one hour. First, she started at the complete opposite end of the virtual world from the virtual spider. Slowly, she got a little closer. In later sessions, after she had lost some of her fear of spiders, she was sometimes encouraged to pick up the virtual spider and/or spider web with her cyber hand and place it in positions that were the most anxiety provoking.

The system application had different levels at which one or more small animals could appear. The animals randomly appeared when the user opened the door of a cupboard. It was possible to kill the animals and dispose of them in dustbin.

\subsection{Augmented reality for psychological treatments}

(Juan et al., 2005) and (Botella et al., 2005) presented the first AR system for the treatment of phobias of cockroaches and spiders. In these works, they demonstrated that, with a single one-hour session, patients significantly reduced their fear and avoidance. Initially, the system was tested in a case study (Botella et al., 2005), and then it was tested on nine patients suffering from phobia towards small animals (Juan et al., 2005). In all cases, the patients reduced their fear and avoidance of the feared animal in only one session of treatment using the VMARS (Suso-Ribera C., Fernández-Álvarez J., 2019). Moreover, all of them were able to interact with the real animal after the treatment. Before the treatment, none of them were able to approach or interact with the live animal without fear. For the treatment of acrophobia, Juan et al. (Juan et al., 2006) proposed the use of immersive photography in an AR system for the treatment of this phobia. In this system, forty-one participants without acrophobia walked around at the top of a staircase in both a real environment and in an immersive photography environment. Immediately after their experience, the participants were given the SUS questionnaire to assess their subjective sense of presence. The users' scores in the immersive photography environment were very high. The results indicated that the acrophobic context could be useful for the treatment of acrophobia. However, statistically significant differences were found between the real and immersive photography environments.

\section{Methodology}

Generally, we focus on optical techniques because the VMARS and the IMARS are marker-based. Optical methods can dived into marker-based/fiducial-based or marker less/natural features-based. The markerbased methods use fiducials that are easily recognizable landmarks or markers such as rectangles, circles, chessboards, etc. These fiducials can be passive (e.g., a printed marker) or active (e.g., a light-emitting diode). Each fiducial consisted of an inner dot and a surrounding outer ring; four different colors use, and thus 12 unique fiducials can create and identify based on their two colors. The work that is most related to ours is the one presented by Park \& Park (2004). They created the invisible markers using an IR fluorescent pen. The system consists of a color camera, an IR camera, and a half mirror. The two cameras position on each side of the half mirror so that their optical centers coincided with each other. They track the invisible marker using the IR camera and visualize AR in the view of the color camera Compare to our system we also use an infrared ink to draw the5markers and two cameras (a color camera and an IR camera), but we do not use the half mirror. Therefore, the hardware characteristics of the two systems are different. 


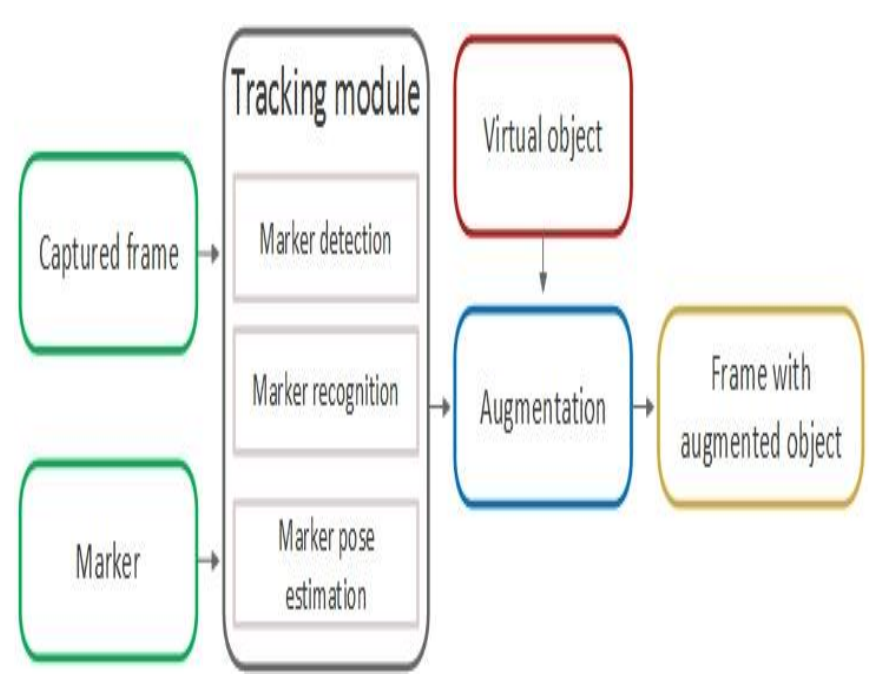

Figure 1. AR Tracking with Marker

\subsection{Hardware}

To capture the video, SAMSUNG galaxy-s8 feature a 12-megapixel "Dual Pixel" rear-facing camera with a $\mathrm{f} / 1.7$ lens. This camera is shown in Figure 2

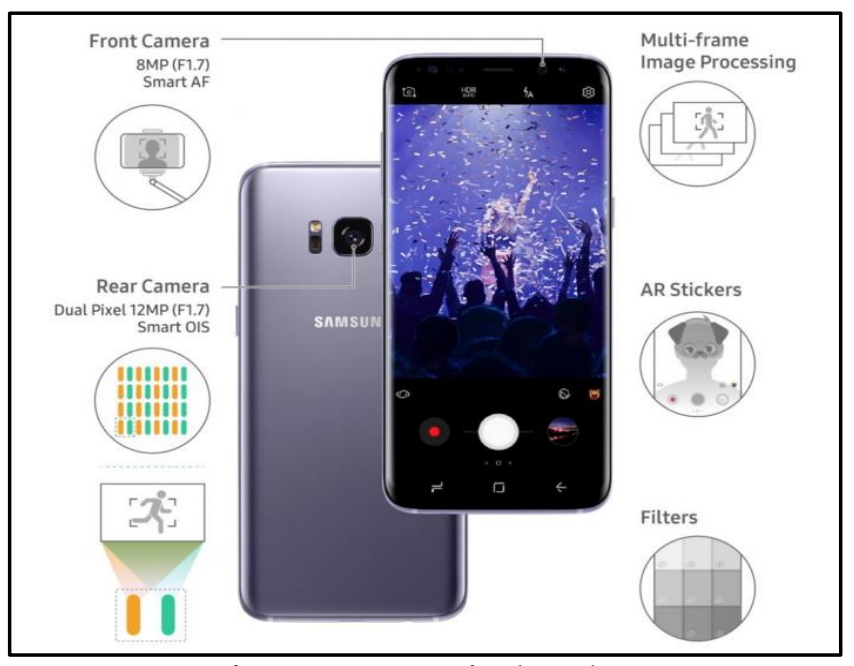

Figure 2. Megapixel Webcam.

Samsung Gear VR is a realistic head-mounted screen developed by Samsung Electronics, in collaboration with Oculus VR, and manufactured by Samsung.

During the use, the Samsung Galaxy-compatible device acts as a headset's display and processor, while the Gear VR itself acts as a controller, which has a field of view, as well as a special inertial measuring module, or IMU, for gyro-tracking, which via USB-C or micro-USB connection with a smartphone.

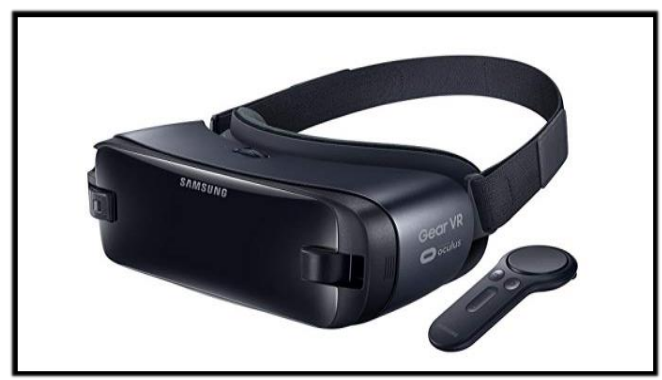

Figure 3. Capture and Visualization System (Webcam, and the HMD)

\subsection{Software}

The functionality of IMARS is the same as VMARS. Therefore, this paper only comments on its functionalities briefly. The therapist/person in charge of the experiment can select the number of lizard to appear: one lizard, increase/reduce by three lizards, or increase/reduce by 2 lizards. We use different size of the lizard / s one of them is small the other is big. The lizards can move or stop. It is possible to kill lizards using two different elements (a can of insecticide and a flyswatter). A detailed description of the functionality of VMARS can be found in Juan et al., 2005).

To develop the two systems, ARCore has been used (Amadeo, Ron, 2017) with virtual reality modeling language (VRML). The three-dimensional models of the virtual elements design using Autodesk Maya. Textures create in 3D Maya. VMARS and IMAR Incorporated three different lizards and three models design: a nonmoving, a moving, and a die lizard. The non-moving lizards shows in static scenes, that is, when the lizards still. The moving models, that is, the model that includes the animation of the lizards, show when the moving option select. The die lizard appears when the user has killed one or two lizards. To obtain a result as real as possible, the moving lizards are animated with moving legs and moving antennae, and the lizards move their legs. The graphical user interface creates 
using Unity video game engine, exporters for Maya and a faster decryption system.

\section{Conclusion}

By using exposure in vivo and virtual reality phobia towards lizards has been treated.

Recently, augmented reality (AR) has also been presented as a suitable tool. The first AR system developed for this purpose used visible markers for tracking. In this first system, the presence of visible markers warns the user of the appearance of lizards. To avoid this warning, this proposal presents a second version in which the markers are invisible. First, the technical characteristics of a prototype are described. Second, a comparative study of the sense of presence and anxiety in a non-phobic population using the visible marker-tracking system and the invisible marker-tracking system is presented. Finally, IMARS and VMARS have the same functionality and performance. As an invisible marker-tracking system, IMARS has two advantages: the user does not see any intruding element in the scene; and the fusion of the virtual objects with the real scene is totally natural. These advantages can be useful not only for psychological applications, but also for any application in which the non-appearance of the marker is important such as applications for art exhibitions or publicity.

\section{References}

1. Suso-Ribera C., Fernández-Álvarez J., 2019. Virtual Reality, Augmented Reality, and In Vivo Exposure Therapy: A Preliminary Comparison of Treatment Efficacy in Small Animal Phobia, 22(1):31-38.

2. Hani Alkurdi, 2019. Augmented Reality Electric Circuit Experiment. 2019 International Conference On Advanced Science And Engineering (ICOASE). doi: 10.1109/icoase.8723683.

3. Milgram and Kishino, 1994. A taxonomy of mixed reality visual displays, IEICE TRANSACTIONS on Information and
Systems Vol.E77-D No.12 pp.1321-1329, Print ISSN: 09168532, Publication .

4. Rothbaum, B. O., Hodges, L., Kooper, R., Opdyke, D., Williford, J. S. \& North, M., 1995. Effectiveness of computer generated (virtual reality) graded exposure in the treatment of acrophobia. American Journal of Psychiatry, 152: 626-628.

5. Bouchard, S., St-Jaques, J., Robillard, G. \& Renaud, P., 2008. Anxiety increases the feeling of presence in virtual reality, Presence: Teleoperators and Virtual Environments, 17(4):376390.

6. Hani Alkurdi, , 2017. "VR Mobile Acrophobia Treatment", Academic Journal of Nawroz University, vol. 6, no. 3, pp. 124-128. Available: 10.25007/ajnu. v6n3a95.

7. Renaud, P., Bouchard, S. \& Proulx, R., 2002. Behavioral avoidance dynamics in the presence of a virtual spider, IEEE Trans. on Information Technology in Biomedicine, 6(3): 235-243.

8. Schuemie, M. J., Bruynzeel, M., Drost, L., Brinckman, M., de Haan, G. \& EmmelKamp, P. M. G., 2000. Treatment of acrophobia in virtual reality: A pilot study. In F. Broeckx \& L. Pauwels (Eds.), Euromedia, 271-275.

9. Botella , C., Baños, R., Quero, S., Perpiña, C. \& Fabregat, S., 2004. TelePsychology and Self-Help: The treatment of phobias using the Internet, Cybertherapy, CD-ROM.

10. Botella, C., Juan, M.C., Baños, R.M., Alcañiz, M., Guillen, V. \& Rey, B., 2005. Mixing realities? An Application of augmented reality for the treatment of cockroach phobia. CyberPsychology \& Behavior, 8: 162-171.

11. Meyerbröker K, Morina N, Kerkhof G, Emmelkamp PM., 2010 . Virtual reality exposure treatment of agoraphobia: a comparison of computer automatic virtual environment and head-mounted display. Journal Article, Randomized Controlled Trial, Research Support, Non-U.S. Gov't, Comparative Study.

12. Discover New Possibilities with the Samsung Galaxy S8 and S8+: Smartphones Without Limits. (2019). from https://news.samsung.com / global/ discover-newpossibilities- with

13. the-samsung-galaxy-s8-a-smartphone-without-limits.

14. Amadeo, Ron, 2017. "Google's ARCore brings augmented reality to millions of Android devices". Ars Technica. Condé Nast. 